\title{
Viel hilft nicht immer viel
}

Fragestellung: Führt ein intensives, strukturiertes und aufgabenspezifisches Training zu einer besseren Rehabilitation der motorischen Handfunktion als eine herkömmliche ambulante Therapie?

Hintergrund: Weder für die spezifische Art noch für die Intensität irgendeiner im ambulanten Alltag praktizierten motorischen Rehabilitation besteht eine ausreichende Evidenz für die Überlegenheit gegenüber einer anderen Therapieform. Bisherige Studienergebnisse legen nahe, dass ein intensives, aufgabenspezifisches Training mit hoher Wiederholungsrate zu einem verbesserten Therapieergebnis führt.

Carolee J, Winstein, Steven L et al. Effect of a task-oriented rehabilitation program on upper extremity recovery following motor stroke. JAMA 2016; 315: $571-81$
Patienten und Methodik: Es handelt sich um eine einfach verblindete randomisierte Studie mit drei parallelen Behandlungsarmen an insge- samt 361 Schlaganfallpatienten mit moderaten Einschränkungen der motorischen Handfunktion. Die eigentliche Behandlungsgruppe erhielt ein intensives, aufgabenspezifisches Trainingsprogramm (Accelerated Skill Acquisition Program, ASAP). Beide Kontrollgruppen erhielten eine herkömmliche Behandlung, einmal 30 Stunden und einmal ohne Vorgabe des Behandlungsumfangs. Primärer Endpunkt war die Veränderung der Handfunktion ermittelt mithilfe des Wolf Motor Function Test (WMFT) nach zwölf Monaten.

Ergebnisse: Die gesamte Studienpopulation zeigte nach zwölf Monaten eine 54\%ige Verbesserung im WMFT. Unterschiede in den drei Behandlungsgruppen waren dabei nur äußerst gering.

Schlussfolgerungen: Schlaganfallpatienten mit moderater Einschränkung der motorischen Handfunktion profitieren nicht weiter von ASAP im Vergleich zu einer dosisäquivalenten oder niederfrequenteren herkömmlichen Behandlung.

\section{- Kommentar von David Liebetanz, Göttingen}

\section{Der Weg zur evidenzbasierten Neurorehabilitation ist schwierig}

Das Evidenzniveau für Verfahren der Neurorehabilitation ist im Allgemeinen sehr begrenzt. Gründe hierfür sind unter anderem ein niedriger Standardisierungsgrad der Behandlungsformen, das Verharren in traditionellen Schulen, die unzureichende Einbindung in die universitäre Ausbildung und Forschung sowie die chronische Unterfinanzierung. Seit Jahren werden vielfältige Bemühungen unternommen, um den Weg von einer empiriegeleiteten zur einer evidenzbasierten Neurorehabilitation einzuschlagen. Speziell werden von den Erkenntnissen der Neurowissenschaften zur Neuroplastizität effektivere und evidenzbasierte Therapien erwartet.

Die nordamerikanischen Autoren haben nun die Effektivität eines neuartigen, intensiven Therapieverfahrens mit herkömmlichen Behandlungsverfahren verglichen, welches in vielen Aspekten auf neurowissenschaftlichen Erkenntnissen beruht. Es zeigte sich jedoch keine Überlegenheit ihres Therapieverfahrens. Mehr noch, selbst mit einem mehr als doppelt so hohen Behandlungsumfang ( 27 versus 11 Stunden) konnte keine Überlegenheit erzielt werden.

Reflexartig stellten sich zwei Fragen: Ist die Patientenzahl ausreichend hoch, um mögliche Unterschiede feststellen zu können? Und: Ist das Testverfahren geeignet, relevante Unterschiede in der Verbesserung darzustellen? Beides kann mit ja beantwortet werden. Ein Umstand, der folgerichtig zu einer Publikation der Daten in einem sehr angesehenen Journal geführt hat, obgleich keine Überlegenheit gezeigt werden konnte.

Womöglich liegt aber auch ein Deckeneffekt vor. Hierauf weisen die $54 \%$ Verbesserung über alle Behandlungsgruppen in der Studienpopulation mit moderaten motorischen Einschränkungen hin (Median des NIH Stroke Scale Score =4). Allein elf Stunden herkömmliche Behandlung führten in dieser hochselektierten Population bereits zu einer relevanten Verbesserung der Handfunktion. Die Verdopplung des Behandlungsumfangs sowie die Einbindung neurowissenschaftlicher Konzepte konnte daher keine Verbesserung darüber hinaus bewirken. Vielleicht hätten bereits fünf Stunden ASAP ausgereicht, um einen gleichartigen Therapieeffekt zu erreichen? Es wäre dann jedoch eher eine ökonomische Überlegenheit als eine absolute therapeutische.

Fazit: Der notwendige Weg hin zu einer evidenzbasierten Neurorehabilitation ist aufwändig und schwierig. Dafür führt er uns jedoch zu neuen Erkenntnissen, wie auch im Falle der hier vorgestellten Studie: Viel hilft nicht immer viel!

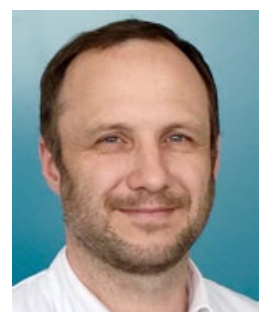

Prof. Dr. med. David Liebetanz, Göttingen

Universitätsmedizin Göttingen Klinik für Klinische Neurophysiologie E-Mail: clneuphy@gwdg.de 